\title{
New robust confidence intervals for the mean under dependence
}

\author{
Martial Longla and Magda Peligrad \\ Department of Mathematics, University of Mississippi, University, MS 38677, \\ USA. E-mail: mlongla@olemiss.edu \\ and \\ Department of Mathematical Sciences, University of Cincinnati, PO Box \\ 210025, Cincinnati, Oh 45221-0025, USA. Email:peligrm@ucmail.uc.edu \\ Key words: Nadaraya-Watsom estimator, central limit theorem, long mem- \\ ory, confidence intervals \\ Mathematical Subject Classification (2000): 60F05, 60F17, 62G08, 62G15.
}

\begin{abstract}
The goal of this paper is to indicate a new method for constructing normal confidence intervals for the mean, when the data is coming from stochastic structures with possibly long memory, especially when the dependence structure is not known or even the existence of the density function. More precisely we introduce a random smoothing suggested by the kernel estimators for the regression function. Applications are presented to linear processes and reversible Markov chains with long memory.
\end{abstract}

\section{Introduction and results}

Let us suppose that we have a stationary and ergodic sequence $\left(Y_{i}\right)_{i \in Z}$ with finite variance $\left(\operatorname{var}\left(Y_{0}\right)=\sigma_{Y}^{2}<\infty\right)$. Denote by $\mu_{Y}=E Y_{0}$, the expected value of $Y$. Also, denote as usual the sample mean by

$$
\bar{Y}_{n}=\frac{1}{n} \sum_{i=1}^{n} Y_{i}=\frac{1}{n} S_{n}^{Y} .
$$

By the Birkhoff ergodic theorem it is well-known that

$$
\lim _{n \rightarrow \infty} \bar{Y}_{n}=\mu_{Y} .
$$

If the sequence $\left(Y_{i}\right)_{i \in Z}$ has short range dependence and we have additional information on its dependence structure, such as martingale-like conditions or mixing conditions, we can derive a central limit theorem for $\sqrt{n}\left(\bar{Y}_{n}-\mu_{Y}\right)$, which naturally leads to the construction of confidence intervals for $\mu_{Y}$ based on normal distribution scalars. Without other information on the dependence structure of $\left(Y_{i}\right)_{i \in Z}$, obviously, such a sequence might not obey the central limit 
theorem, and this method is not possible to use. In this note we indicate a way to construct normal confidence intervals for $\mu_{Y}$ based on a smoothing method inspired by Nadaraya-Watson estimators.

For the purpose of this paper, we shall say that a sequence $\left(Y_{i}\right)_{i \in Z}$ has long range dependence if $\operatorname{var}\left(S_{n}^{Y}\right) / n \rightarrow \infty$ and short range if $\operatorname{var}\left(S_{n}^{Y}\right)$ behaves linearly in $n$.

Given a sample $\left(X_{i}, Y_{i}\right)_{1 \leq i \leq n}$ from a random vector $(X, Y)$ on a probability space $(\Omega, K, P)$, the well-known Nadaraya-Watson estimator (see Nadaraya (1964) and Watson (1964), or pages 126-127 in Härdle (1991)) is defined by

$$
\hat{m}_{n}(x)=\frac{1}{n h_{n} \hat{f}_{n}(x)} \sum_{i=1}^{n} Y_{i} K\left(\frac{1}{h_{n}}\left(X_{i}-x\right)\right),
$$

where

$$
\hat{f}_{n}(x)=\frac{1}{n h_{n}} \sum_{i=1}^{n} K\left(\frac{1}{h_{n}}\left(X_{i}-x\right)\right) .
$$

This estimator has been widely studied in the literature. For instance, when the vector $(X, Y)$ has joint density $f(x, y)$ say, $\hat{m}_{n}(x)$ is used to estimate

$$
E(Y \mid X=x)=r(x)=\int y[f(x, y) / f(x)] d y .
$$

Furthermore, when $K$ is a kernel with several properties, $h_{n}$ is a sequence of positive numbers (bandwidth) such that

$$
h_{n} \rightarrow 0 \text { and } n h_{n} \rightarrow \infty \text { as } n \rightarrow \infty,
$$

under various smoothness assumptions on $(X, Y)$ and various dependence assumptions on the process $\left(X_{i}, Y_{i}\right)_{i \in Z}$, the speed of convergence of $\hat{m}_{n}(x)$ to $r(x)$ was pointed out in numerous papers. The dependence structure considered in the literature is rather restrictive, of the weak dependence type, such as mixing conditions, function of mixing sequences or martingale-like conditions. We mention for instance results in Bradley (1983), Collomb (1984), Peligrad (1992), Yoshihara (1994), Bosq (1996), Bosq et.al. (1999), Long and Qian (2013), and Hong and Linton (2016) among many others.

Now, let us notice that if the variables $\left(X_{i}\right)_{i \in Z}$ are independent of $\left(Y_{i}\right)_{i \in Z}$, we have $E(Y \mid X)=E(Y)=\mu_{Y}$. Inspired by Nadaraya-Watson estimator, the goal of our paper is to indicate how this observation can be used to develop robust procedures for constructing normal interval estimates for the mean $\mu_{Y}$ by using the estimator

$$
\hat{m}_{n}(0)=\frac{1}{n h_{n} \hat{f}_{n}(0)} \sum_{i=1}^{n} Y_{i} K\left(\frac{1}{h_{n}} X_{i}\right),
$$

when there is very little information about the dependence structure of the sequence $\left(Y_{i}\right)_{i \in Z}$ or the existence of the density of $Y$. 
The procedure we propose is the following. The data $\left(Y_{i}\right)_{1 \leq i \leq n}$ consists of a sample from a stationary and ergodic sequence $\left(Y_{i}\right)_{i \in Z}$. Independently of $\left(Y_{i}\right)_{1 \leq i \leq n}$ we generate a random sample $\left(X_{i}\right)_{1 \leq i \leq n}$, from a distribution with bounded density $f(x)$, continuous at the origin, with $f(0) \neq 0$. It is known that $\hat{f}_{n}(0)$ is an asymptotically unbiased estimator for $f(0)$, provided the bandwidths $h_{n}$ satisfies the condition (11) and the kernel $K$ satisfies (see Parzen, 1962 or Härdle, page 59) the following condition

$K$ is a symmetric bounded density function.

Under these conditions, $\lim _{n \rightarrow \infty} \hat{f}_{n}(0)=f(0)$ in $L_{2}$. Therefore, by Slutsky's theorem, we can replace the study of the limiting distribution of $\hat{m}_{n}$ by that of its asymptotic equivalent estimator

$$
\hat{r}_{n}=\frac{1}{n h_{n} f(0)} \sum_{i=1}^{n} Y_{i} K\left(\frac{1}{h_{n}} X_{i}\right) .
$$

The estimator $\hat{m}_{n}(0)$ is an unbiased estimator of $\mu_{Y}$, while $\hat{r}_{n}$ is asymptotically unbiased. However $\hat{m}_{n}(0)$ has the disadvantage that it introduces an error due to replacing the known quantity $f(0)$ by its estimate $\hat{f}_{n}(0)$. In addition $\hat{r}_{n}$ is easier to analyze. This is the reason why we prefer to use $\hat{r}_{n}$ as our proposed estimator for $\mu_{Y}$. We shall provide a central limit theorem, a functional central limit theorem and also discuss the optimal bandwidth which minimizes the mean square error.

To establish these results, we use the independence structure of the smoothing sequence $\left(X_{i}\right)_{i \in Z}$ that allows us not to restrict the dependence structure of $\left(Y_{i}\right)_{i \in Z}$ and also not to impose the existence of the density of $Y$. The closest idea to this one is the block-wise bootstrap. For instance in the paper by Peligrad (1998), the central limit theorem for the mean is obtained via bootstrap smoothing, for a sequence that does not satisfy the CLT, but rather satisfies some restrictive mixing conditions. In the sequel we denote by $\Rightarrow$ the convergence in distribution. For positive sequences of numbers $a_{n}=O\left(b_{n}\right)$ means $\limsup _{n \rightarrow \infty} a_{n} / b_{n}<\infty ; a_{n}=o\left(b_{n}\right)$ means $\lim _{n \rightarrow \infty} a_{n} / b_{n}=0$. We use the notation $a_{n} \sim b_{n}$ for $\lim _{n \rightarrow \infty} a_{n} / b_{n}=1$.

Besides condition (1) we shall impose the following assumption on the bandwidths sequence $\left(h_{n}\right)_{n \geq 1}$ :

$$
\sqrt{n h_{n}}\left(\bar{Y}_{n}-\mu_{Y}\right) \rightarrow^{P} 0
$$

which is implied by

$$
n h_{n} \operatorname{var}\left(\bar{Y}_{n}\right) \rightarrow 0 .
$$

Note that we can always find a sequence $\left(h_{n}\right)_{n \geq 1}$ satisfying both conditions (1) and (5), provided that $\operatorname{var}\left(\bar{Y}_{n}\right) \rightarrow 0$.

We shall establish the following theorem: 
Theorem 1 Assume that $\left(Y_{i}\right)_{i \in Z}$ is a stationary and ergodic sequence with finite second moments and conditions (1) and condition (4) are satisfied. Also assume that $K$ satisfies condition (2) and that $\left(X_{i}\right)_{i \in N}$ is an i.i.d. sequence of random variables, independent of $Y$, having a bounded density function $f(x)$, continuous at the origin, with $f(0) \neq 0$. Then we have

$$
\frac{\sqrt{n h_{n}}}{\sqrt{\overline{Y_{n}^{2}}}}\left(\hat{r}_{n}-\mu_{Y}\right) \Rightarrow N\left(0, \frac{1}{f(0)} \int K^{2}(x) d x\right) .
$$

where $\overline{Y_{n}^{2}}=\sum_{i=1}^{n} Y_{i}^{2} / n$ and $\hat{r}_{n}$ is defined by (3).

By combining this theorem with the consistency of $\hat{f}_{n}(0)$ we obtain that

Corollary 2 Under the conditions of Theorem 11 we also have

$$
\frac{\sqrt{n h_{n} \hat{f}_{n}(0)}}{\sqrt{\overline{Y_{n}^{2}}}}\left(\hat{m}_{n}-\mu_{Y}\right) \Rightarrow N\left(0, \int K^{2}(x) d x\right) .
$$

Based on Theorem 1 we can construct confidence intervals for the mean:

Corollary 3 Under the conditions of Theorem 1, for $0<\alpha<1$, $a(1-\alpha) 100 \%$ confidence interval for $\mu_{Y}$ is

$$
\left\{\hat{r}_{n}-z_{\alpha / 2}\left(\frac{\overline{Y_{n}^{2}} \int K^{2}(x) d x}{n h_{n} f(0)}\right)^{1 / 2}, \hat{r}_{n}+z_{\alpha / 2}\left(\frac{\overline{Y_{n}^{2}} \int K^{2}(x) d x}{n h_{n} f(0)}\right)^{1 / 2}\right\},
$$

where $P\left(-z_{\alpha / 2}<Z<z_{\alpha / 2}\right)=1-\alpha$ and $Z$ is a standard normal variable.

Let us notice that, at no extra cost, our result can be also formulated as a functional CLT. If we consider the stochastic process

$$
\hat{r}_{n}(t)=\frac{1}{n h_{n} f(0)} \sum_{i=1}^{[n t]} Y_{i} K\left(\frac{1}{h_{n}} X_{i}\right), \hat{m}_{n}(t)=\frac{1}{n h_{n} \hat{f}(0)} \sum_{i=1}^{[n t]} Y_{i} K\left(\frac{1}{h_{n}} X_{i}\right),
$$

from the proof of Theorem 1 and Donsker's theorem (see Theorem 8.2 in Billingsley, 1999) we obtain:

Corollary 4 Under the conditions of Theorem 11 we have

$$
\sqrt{n h_{n}}\left(\hat{r}_{n}(t)-\mu_{Y}\right) / \sqrt{\overline{Y_{n}^{2}}} \Rightarrow\left(\frac{1}{f(0)} \int K^{2}(x) d x\right)^{1 / 2} W(t),
$$

where $W(t)$ is the standard Brownian motion, and also

$$
\sqrt{n h_{n} \hat{f}_{n}(0)}\left(\hat{m}_{n}(t)-\mu_{Y}\right) / \sqrt{\overline{Y_{n}^{2}}} \Rightarrow\left(\int K^{2}(x) d x\right)^{1 / 2} W(t) .
$$

Our paper is organized as follows: In Section 2 we prove Theorem 1 . In Section 3 we discuss the data driven selection of the optimal bandwidth to be used in confidence intervals. Several applications to processes with long memory are given in Section 4. In the last section we mention several remarks. 


\section{Proof of Theorem 1}

For convenience, we shall drop the index $n$ from the notation of $h_{n}$. We condition on $\left(Y_{i}\right)_{i \in Z}$ and we shall first find the limiting distribution of a related sequence of random variables under the regular conditional probability $P_{Y}^{\omega}(\cdot)=$ $P\left(\cdot \mid\left(Y_{i}\right)_{i \in Z}\right)(\omega)$. In the sequel $E_{Y}^{\omega}$ denotes the expected value with respect to $P_{Y}^{\omega}$. We introduce the sequence of random variables

$$
Z_{n, i}=\frac{1}{\sqrt{h}}\left(K\left(\frac{1}{h} X_{i}\right)-E\left(K\left(\frac{1}{h} X_{i}\right)\right)\right) Y_{i}=X_{n, i} Y_{i}
$$

where

$$
X_{n, i}=\frac{1}{\sqrt{h}}\left[K\left(\frac{1}{h} X_{i}\right)-E\left(K\left(\frac{1}{h} X_{i}\right)\right)\right] .
$$

Note that, by the independence of sequences $\left(Y_{i}\right)_{i \in Z}$ and $\left(X_{i}\right)_{i \in Z}$, for almost all $\omega$, we have

$$
E_{Y}^{\omega}\left(Z_{n, i}\right)=Y_{i}(\omega) E\left(X_{n, i}\right)=0 .
$$

Denote

$$
W_{n}=\frac{1}{\sqrt{n}} \sum_{i=1}^{n} Z_{n, i}=\frac{1}{\sqrt{n}} \sum_{i=1}^{n} X_{n, i} Y_{i} .
$$

Let us find the limiting distribution of $W_{n}$ under $P_{Y}^{\omega}$, for almost all $\omega$. We start by constructing $\Omega^{\prime}$ such that, for all $\omega \in \Omega^{\prime}$ the following convergences hold:

$$
\lim _{n \rightarrow \infty} \frac{1}{n} \sum_{i=1}^{n} Y_{i}^{2}(\omega)=E\left(Y^{2}\right)
$$

and for all $A$, positive integer

$$
\lim _{n \rightarrow \infty} \frac{1}{n} \sum_{i=1}^{n} Y_{i}^{2}(\omega) I\left(\left|Y_{i}\right|(\omega)>A\right)=E\left[Y^{2} I(|Y|>A)\right] .
$$

This is possible because $\left(Y_{i}\right)_{i \in Z}$ is ergodic, so the convergences in (8) and (9) hold on sets of measure 1 . We construct $\Omega^{\prime}$ as a countable intersection of these sets, which will also have measure 1 . Fix $\omega \in \Omega^{\prime}$.

Under $P_{Y}^{\omega},\left(W_{n}\right)_{n \geq 1}$ becomes a sum of a triangular array of independent random variables. Therefore, in order the establish the CLT, we have to take care of the limiting variance and then verify the Lindeberg's condition. All the integrals below are taken over $R=(-\infty, \infty)$.

First we recall that for all $i \in N$,

$$
\begin{aligned}
\operatorname{var}\left(X_{n, i}\right) & =\frac{1}{h} \int K^{2}\left(\frac{t}{h}\right) f(t) d t-\frac{1}{h}\left(\int K\left(\frac{t}{h}\right) f(t) d t\right)^{2} \\
& =\int K^{2}(t) f(t h) d t-h\left(\int K(t) f(t h) d t\right)^{2} .
\end{aligned}
$$


So, by Bochner's theorem and condition (2),

$$
\lim _{n \rightarrow \infty} \operatorname{var}\left(X_{n, i}\right)=\lim _{n \rightarrow \infty} E\left(X_{n, i}^{2}\right)=f(0) \int K^{2}(u) d u=C_{1} .
$$

By the independence of sequences $\left(Y_{i}\right)_{i \in Z}$ and $\left(X_{i}\right)_{i \in Z}$ and stationarity we have

$$
\sigma_{n}^{2}(\omega)=\operatorname{var}_{Y}^{\omega}\left(W_{n}\right)=\frac{1}{n} \sum_{i=1}^{n} Y_{i}^{2}(\omega) \operatorname{var}\left(X_{n, 1}\right)=\overline{Y_{n}^{2}}(\omega) \operatorname{var}\left(X_{n, 1}\right)
$$

and therefore, by (8)

$$
\lim _{n \rightarrow \infty} \sigma_{n}^{2}(\omega)=\lim _{n \rightarrow \infty} \frac{C_{1}}{n} \sum_{i=1}^{n} Y_{i}^{2}(\omega)=C_{1} E\left(Y^{2}\right) .
$$

Let us establish now the Lindeberg's condition under $P_{Y}^{\omega}$.

Denote $\sigma_{n}(\omega)=\sqrt{\sigma_{n}^{2}(\omega)}$. We have to show that, for every $\varepsilon>0$,

$$
\lim _{n \rightarrow \infty} \frac{1}{\sigma_{n}(\omega)} \frac{1}{n} \sum_{i=1}^{n} E_{Y}^{\omega}\left[X_{n, i}^{2} Y_{i}^{2} I\left(\left|X_{n, i} Y_{i}\right| \geq \varepsilon \sigma_{n}(\omega) \sqrt{n}\right)\right]=0 .
$$

Now, by (11) there is $N(\varepsilon, \omega)$ such that for all $n>N(\varepsilon, \omega)$ we have $\sigma_{n}(\omega) \geq$ $C_{1} E\left(Y^{2}\right) / 2$. By this remark, by the independence of the two sequences (see Example 33.7 in Billingsley) and stationarity, we obtain

$$
E_{Y}^{\omega}\left[X_{n, i}^{2} Y_{i}^{2} I\left(\left|X_{n, i} Y_{i}\right| \geq \varepsilon \sigma_{n}(\omega) \sqrt{n}\right)\right]=Y_{i}^{2}(\omega) E\left[X_{n, 1}^{2} I\left(\left|X_{n, 1} Y_{i}(\omega)\right| \geq \varepsilon \sigma_{n}(\omega) \sqrt{n}\right)\right] .
$$

It follows that, in order to show (12), we have to show instead

$$
\lim _{n \rightarrow \infty} \frac{1}{n} \sum_{i=1}^{n} Y_{i}^{2}(\omega) E\left[X_{n, 1}^{2} I\left(\left|X_{n, 1} Y_{i}(\omega)\right| \geq \varepsilon^{\prime} \sqrt{n}\right)\right]=0,
$$

where we denoted $\varepsilon^{\prime}=\varepsilon C_{1} E\left(Y^{2}\right) / 2$. Denote the expression above:

$$
G_{n}(\omega)=\frac{1}{n} E\left[X_{n, 1}^{2} \sum_{i=1}^{n} Y_{i}^{2}(\omega) I\left(\left|X_{n, 1} Y_{i}(\omega)\right| \geq \varepsilon^{\prime} \sqrt{n}\right)\right] .
$$

We shall decompose the sum in two parts. Let $A$ be a positive integer and define the index sets

$$
\begin{aligned}
& I_{1}(\omega)=\left(i: 1 \leq i \leq n,\left|Y_{i}\right|(\omega) \leq A\right), \\
& I_{2}(\omega)=\left(i: 1 \leq i \leq n,\left|Y_{i}\right|(\omega)>A\right) .
\end{aligned}
$$

Note $\{1,2, \ldots, n\}=I_{1}(\omega) \cup I_{2}(\omega)$. We write $\sum_{i=1}^{n}=\sum_{i \in I_{1}(\omega)}+\sum_{i \in I_{1}(\omega)}$ and, by using the stationarity assumption, we shall upper bound $F_{n}$ in the following way:

$G_{n}(\omega) \leq A^{2} E\left[X_{n, 1}^{2} I\left(\left|X_{n, 1}\right| \geq A^{-1} \varepsilon^{\prime} \sqrt{n}\right)\right]+E\left(X_{n, 1}^{2}\right) \frac{1}{n} \sum_{i=1}^{n} Y_{i}^{2}(\omega) I\left(\left|Y_{i}\right|(\omega)>A\right)$. 
Note that :

$$
\begin{gathered}
E\left[X_{n, 1}^{2} I\left(\left|X_{n, 1}\right| \geq A^{-1} \varepsilon^{\prime} \sqrt{n}\right)\right]=\frac{1}{h} \int K^{2}\left(\frac{v}{h}\right) I\left(K\left(\frac{v}{h}\right) \geq A^{-1} \varepsilon^{\prime} \sqrt{n h}\right) f(v) d v= \\
\int K^{2}(u) I\left(K(u) \geq A^{-1} \varepsilon^{\prime} \sqrt{n h}\right) f(u h) d u .
\end{gathered}
$$

Since $n h \rightarrow \infty$ and $K$ is bounded, this limit is 0 as $n \rightarrow \infty$. By passing to the limit in (13) with $n \rightarrow \infty$ and by using (10), we easily obtain

$$
\lim \sup _{n \rightarrow \infty} G_{n}(\omega)=C_{1} E\left[Y^{2} I(|Y|>A)\right] .
$$

By letting $A \rightarrow \infty$, and using the fact that $Y$ has finite second moment, we get

$$
\lim _{n \rightarrow \infty} G_{n}(\omega)=0 .
$$

Therefore, the Lindeberg's condition is satisfied under $P_{Y}^{\omega}$. By all this considerations, we obtain that the following quenched central limit theorem holds: for any fixed $\omega \in \Omega^{\prime}$

$$
W_{n} \Rightarrow N\left(0, C_{1} E\left(Y^{2}\right)\right) \text { under } P_{Y}^{\omega} \text {. }
$$

This quenched CLT is a stronger form of CLT. After representing it in terms of characteristic function we can integrate with respect to the measure $P$ and we obtain the annealed CLT, namely

$$
W_{n} \Rightarrow N\left(0, C_{1} E\left(Y^{2}\right)\right) \text { under } P .
$$

Now recall the definition of $Z_{n, i}=h^{-1 / 2}\left(K\left(\frac{1}{h} X_{i}\right)-E\left(K\left(\frac{1}{h} X_{i}\right)\right)\right) Y_{i}$. Let us also note that by definition (3),

$$
\frac{1}{\sqrt{n h}} \sum_{i=1}^{n} Y_{i} K\left(\frac{1}{h} X_{i}\right)=\sqrt{n h} f(0) \hat{r}_{n}
$$

So we can rewrite

$$
W_{n}=\sqrt{n h}\left(f(0) \hat{r}_{n}-\frac{1}{n h} \sum_{i=1}^{n} Y_{i} E\left[K\left(\frac{1}{h} X_{i}\right)\right]\right) .
$$

Note that, by the properties of $K$,

$$
\lim _{n \rightarrow \infty} \sqrt{n h}\left(\frac{1}{n h} \sum_{i=1}^{n} Y_{i} E\left(K\left(\frac{1}{h} X_{i}\right)\right)-f(0) \mu_{Y}\right)=\lim _{n \rightarrow \infty} \sqrt{n h} f(0)\left(\frac{1}{n} \sum_{i=1}^{n} Y_{i}-\mu_{Y}\right) .
$$

If we impose (5), then

$$
\sqrt{n h}\left(\frac{1}{n h} \sum_{i=1}^{n} Y_{i} E\left(K\left(\frac{1}{h} X_{i}\right)\right)-f(0) \mu_{Y}\right) \rightarrow^{P} 0
$$

and, by Theorem 25.2 in Billingsley (1995), we obtain

$$
\sqrt{n h} f(0)\left(\hat{r}_{n}-\mu_{Y}\right) \Rightarrow N\left(0, C_{1} E\left(Y^{2}\right)\right) .
$$

By the ergodic theorem and Slutski's theorem we obtain the desired result. 


\section{Data driven bandwidth selection.}

The method we propose introduces new parameters, the bandwidth sequence $\left(h_{n}\right)_{n \geq 1}$. There is a vast literature on the selection of $h_{n}$ for kernel estimation of the density and for the Nadaraya-Watson estimator of a regression, under independence or weak dependence assumptions. They can be found in books, such as in Section 5.1.2 in Härdle (1990) or in surveys, such as Jones et. al. (1996). Our case deals with possible long dependence for $\left(Y_{i}\right)_{i \in Z}$ but it benefits from the independence of $\left(Y_{i}\right)_{i \in Z}$ and $\left(X_{i}\right)_{i \in Z}$ and also from the fact that we know $f(x)$. If we impose additional conditions on the smoothness of $f(x)$ and $K(x)$, namely $f(x)$ has a continuous and bounded second derivative and $K$ satisfies condition (2) and $\int u^{2} K(u) d u<\infty$, we can analyze the optimal bandwidth by optimizing the main part of the mean square error under the constraint (5). We shall see that this selection depends on the strength of dependence of $\left(Y_{i}\right)_{i \in Z}$. As a matter of fact we shall prove that

Proposition 5 Under the conditions above, the optimal data driven bandwidth to be used in the confidence intervals is

$$
h_{o}=\left[\frac{f(0) B \overline{Y_{n}^{2}}}{n(f "(0) A)^{2}\left(\bar{Y}_{n}\right)^{2}}\right]^{1 / 5} \text { provided that } \operatorname{var}\left(\bar{Y}_{n}\right)=o\left(n^{-4 / 5}\right) \text { and } \mu_{Y} \neq 0 \text {. }
$$

Proof. Denote $V_{n, i}=h^{-1} K\left(X_{i} / h\right)$. We shall compute first the bias

$$
\operatorname{Bias}\left(\hat{r}_{n}\right)=E\left(\hat{r}_{n}-\mu_{Y}\right)=\frac{\mu_{Y}}{f(0)} E\left(\hat{f}_{n}(0)\right)-\mu_{Y}=\frac{\mu_{Y}}{f(0)} \operatorname{Bias} \hat{f}_{n}(0) .
$$

The variance of the estimator is

$$
\begin{aligned}
\operatorname{var}\left(\hat{r}_{n}\right) & =E\left[\hat{r}_{n}-\frac{\mu_{Y}}{f(0)} E\left(\hat{f}_{n}(0)\right)\right]^{2} \\
& =E\left[\hat{r}_{n}-\frac{\mu_{Y}}{f(0)} \hat{f}_{n}(0)+\frac{\mu_{Y}}{f(0)}\left(\hat{f}_{n}(0)-E\left(\hat{f}_{n}(0)\right)\right]^{2}\right. \\
& =E\left[\hat{r}_{n}-\frac{\mu_{Y}}{f(0)} \hat{f}_{n}(0)\right]^{2}+\frac{\mu_{Y}^{2}}{f^{2}(0)} \operatorname{var} \hat{f}_{n}(0)=I+I I .
\end{aligned}
$$


A simple computation shows that the first term

$$
\begin{gathered}
I=\frac{1}{f^{2}(0)} E\left(\frac{1}{n} \sum_{i=1}^{n}\left(Y_{i}-\mu_{Y}\right) V_{n, i}\right)^{2} \\
=\frac{1}{f^{2}(0) n^{2}}\left[n \sigma_{Y}^{2} E\left(V_{n, i}^{2}\right)+2 \sum_{1 \leq i<j \leq n} \operatorname{cov}\left(Y_{i}, Y_{j}\right)\left(E V_{n, i}\right)^{2}\right] \\
\frac{1}{f^{2}(0) n^{2}}\left[n \sigma_{Y}^{2} E\left(V_{n, i}^{2}\right)+\left(E V_{n, 1}\right)^{2}\left(\operatorname{var} S_{Y}-n \sigma_{Y}^{2}\right)\right]= \\
\frac{1}{f^{2}(0) n^{2}}\left[n \sigma_{Y}^{2} \operatorname{var} V_{n, i}+\left(E V_{n, 1}\right)^{2} \operatorname{var} S_{Y}\right] \\
=\frac{1}{f^{2}(0)}\left[\sigma_{Y}^{2} \operatorname{var} \hat{f}_{n}(0)+\left(E V_{n, 1}\right)^{2} \operatorname{var} \bar{Y}_{n}\right] .
\end{gathered}
$$

Therefore, by combining these estimates, the mean square error is

$$
\begin{aligned}
\operatorname{MSE}\left(\hat{r}_{n}\right) & =E\left(\hat{r}_{n}-\mu_{Y}\right)^{2}=\operatorname{var} \hat{r}_{n}+\left[\operatorname{Bias}\left(\hat{r}_{n}\right)\right]^{2} \\
& =\frac{1}{f^{2}(0)}\left[E\left(Y^{2}\right) \operatorname{var} \hat{f}_{n}(0)+\left(E V_{n, 1}\right)^{2} \operatorname{var} \bar{Y}_{n}+\mu_{Y}^{2}\left[\operatorname{Bias} \hat{f}_{n}(0)\right]^{2} .\right.
\end{aligned}
$$

Under the assumption (5),

$$
\left(E V_{n, 1}\right)^{2} \operatorname{var} \bar{Y}_{n}=o\left(\frac{1}{n h_{n}}\right) .
$$

When $f(x)$ has a continuous and bounded second derivative, according to formula (2.3.2) in Härdle (1990) we have

$$
\operatorname{Bias} \hat{f}_{n}(0)=\frac{h^{2}}{2} f^{\prime \prime}(0) A+o\left(h^{2}\right) \text { as } n \rightarrow \infty,
$$

where

$$
A=\int x^{2} K(x) d x
$$

Also by formula (2.3.3) in the same book

$$
\operatorname{var} \hat{f}_{n}(0)=\frac{1}{n h} B f(0)+o\left(\frac{1}{n h}\right) \text { as } n \rightarrow \infty,
$$

where

$$
B=\int K^{2}(x) d x
$$

It follows that

$$
\operatorname{MSE}\left(\hat{r}_{n}\right)=\frac{1}{f^{2}(0)}\left[\frac{E\left(Y^{2}\right)}{n h} B f(0)+\frac{h^{4}}{4} \mu_{Y}^{2}\left(f^{\prime \prime}(0) A\right)^{2}+o\left(\frac{1}{n h}\right)+o\left(h^{4}\right)\right] .
$$


In order to minimize it, we set 0 the derivative with respect to $h$ of the main part and obtain

$$
h_{o^{\prime}}=\left[\frac{f(0) B E\left(Y^{2}\right)}{n\left(f^{\prime \prime}(0) A\right)^{2} \mu_{Y}^{2}}\right]^{1 / 5},
$$

provided $\mu_{Y} \neq 0$. Since the optimal $h_{o^{\prime}}$ depends on the unknown parameters $E\left(Y^{2}\right)$ and $\mu_{Y}^{2} \neq 0$, we shall replace them by plug in estimators which are consistent because of the ergodicity of $\left(Y_{n}\right)_{n}$ we obtain (17).

Remark 6 This $h_{o}$ was obtained by imposing condition (5). At the same time $h_{o}$ has to satisfy (5), leading to the restriction $\operatorname{var}\left(\bar{Y}_{n}\right)=o\left(n^{-4 / 5}\right)$. Otherwise, if $\limsup _{n \rightarrow \infty}\left(n^{4 / 5} \operatorname{var}\left(\bar{Y}_{n}\right)\right) \neq 0$ the MSE is minimized when $h_{n}$ is the largest possible satisfying (1) and (5).

\section{Applications to stationary sequences with long memory}

Example 1. Restriction on the covariance structure. Let us first point an example of a sequence where no restriction of the dependence structure will be assumed, or the distribution of $Y$, except ergodicity and a mild restriction on the covariances.

For a stationary and ergodic sequence of random variables $\left(Y_{k}\right)_{k \in Z}$ with finite second moment, let us assume that $\left|\operatorname{cov}\left(Y_{0}, Y_{k}\right)\right| \sim C\left(k^{-\alpha}\right)$ as $k \rightarrow \infty$ for $\alpha>0$. For $0<\alpha \leq 1$, the covariances are not summable and $\left(Y_{k}\right)_{k \in Z}$ has long memory. Note first that we have

$$
n h \operatorname{var}\left(\bar{Y}_{n}\right) \leq 2 h_{n} \sum_{k=0}^{n}\left|\operatorname{cov}\left(Y_{0} Y_{k}\right)\right|=O\left(h_{n} n^{-\alpha+1}\right) \text { as } n \rightarrow \infty .
$$

Therefore the condition (5) of Theorem 1 holds as soon as $h_{n}=o\left(n^{-1+\alpha}\right)$ and our CLT applies.

We shall see that when $\alpha>0.8$, Theorem 1 can be applied with an optimal $h_{0} \sim C\left(n^{-1 / 5}\right)$, where $C$ is as in formula (17). Indeed, for this range both (18) and (17) are satisfied. If $0<\alpha \leq 0.8$, the MSE will converge to 0 at a rate slower than $n^{-\alpha}$. On the other hand, if we have $\left|\operatorname{cov}\left(Y_{0}, Y_{k}\right)\right| \sim C(\log k)^{-1}$ then $\sum_{k=0}^{n}\left|\operatorname{cov}\left(Y_{0} Y_{k}\right)\right|=O(n / \log n)$. If we take $h_{n}=o(\log n / n)$ Theorem 1 still can be applied. In this case the rate of convergence to 0 of the MSE is slower than $(\log n)^{-1}$ as $n \rightarrow \infty$. This shows that when the memory is very long the rates of convergence can be rather slow, therefore a very large sample size might be necessary.

Example 2. Long memory linear processes. Let $\left(\xi_{j}\right)_{j \in Z}$ be an i.i.d. sequence of random variables, centered with finite second moments. Let $\left(a_{j}\right)_{j \in Z}$ be a sequence of constants We consider the linear process

$$
Y_{k}=\sum_{j=-\infty}^{\infty} a_{k-j} \xi_{j} .
$$


Denote $S_{n}=\sum_{k=1}^{n} Y_{k}$. If $\sum_{i \in \mathbb{Z}} a_{i}^{2}<\infty$, (19) is well defined a.s. and in $L_{2}$. We can write $S_{n}=\sum_{i=-\infty}^{\infty} b_{n i} \xi_{i}$ with

$$
b_{n i}=a_{1-i}+\cdots+a_{n-i}
$$

Using this notation we have $\operatorname{var}\left(S_{n}\right)=\operatorname{var}\left(\xi_{0}^{2}\right) \sum_{i} b_{n i}^{2}$. Then $\operatorname{var}\left(\sqrt{n h}\left(S_{n} / n\right)\right)=$ $h_{n} n^{-1} \sum_{i} b_{n i}^{2}$.

If we assume (11) and that $h_{n} n^{-1} \sum_{i} b_{n i}^{2} \rightarrow 0$, then the conclusion of Theorem 1 holds.

As a particular example we consider the important case of causal longmemory processes with

$$
a_{i}=[l(i+1)](1+i)^{-\alpha}, i \geq 0, \text { with } 1 / 2<\alpha<1 \text {, and } a_{i}=0 \text { otherwise. }
$$

Here $l(\cdot)$ is a slowly varying function at infinite. These processes have long memory because $\sum_{j \geq 0}\left|a_{j}\right|=\infty$.

For this case, $\operatorname{var}\left(\bar{Y}_{n}\right) \sim \kappa_{\alpha} n^{1-2 \alpha} \ell^{2}(n)$ (see for instance Relations (12) in Wang et al. (2003)), where $\kappa_{\alpha}$ is a positive constant depending on $\alpha$. Theorem 1 can be applied as soon as $h_{n}=o\left(n^{2(1-\alpha)} \ell^{2}(n)\right)^{-1}$ as $n \rightarrow \infty$. In the range $0.9<\alpha<1, h_{o}$ can be taken as in (17). Otherwise the MSE will converge to 0 at a rate slower than $\ell^{2}(n) / n^{2 \alpha-1}$ as $n \rightarrow \infty$.

This example covers the ARFIMA $(0, d, 0)$ processes (cf. Granger and Joyeux (1980); Hosking (1981)), which play an important role in financial time series modeling and application. As a special case, let $0<d<1 / 2$ and $B$ be the backward shift operator with $B \varepsilon_{k}=\varepsilon_{k-1}$,

$$
X_{k}=(1-B)^{-d} \xi_{k}=\sum_{i \geq 0} a_{i} \xi_{k-i}, \text { where } a_{i}=\frac{\Gamma(i+d)}{\Gamma(d) \Gamma(i+1)}
$$

Here $\lim _{n \rightarrow \infty} a_{n} / n^{d-1}=1 / \Gamma(d)$. For this case $\operatorname{var}\left(\bar{Y}_{n}\right) \sim \kappa_{d} n^{2 d-1}$ and condition (17) becomes $h_{n}=o\left(n^{-2(1-d)}\right)$. For this case $h_{o}$ can taken as in (17) for $0<$ $d<0.1$. For $0.1 \leq d<1 / 2$, and a selection of $h_{n}=o\left(n^{-2 / d}\right)$, the MSE will converge to 0 at a rate slower than the order $n^{2 d-1}$ as $n \rightarrow \infty$.

Example 3. A long memory reversible Markov chain. For a nonlinear example we would like to mention an example given in Zhao et al. (2010), describing a stationary and ergodic reversible Markov chain, which does not satisfy the CLT. This is their Example 2. Let $1<\alpha<2$. One starts with a measurable function $p: R \rightarrow(0,1)$ and a probability measure $v$ such that for $|x|>1$

$$
v(x)=\frac{[1-p(x)] d x}{2 \gamma_{\alpha}|x|^{\alpha}} \text { where } \gamma_{\alpha}=\int_{0}^{1} y^{\alpha-2}\left(1-\mathrm{e}^{-y}\right) d y .
$$

We define now a stationary and reversible Markov chain, $\left(X_{n}\right)_{n \in Z}$, with transition operator:

$$
Q(x, A)=p(x) \delta_{x}(A)+(1-p(x)) v(A)
$$


where $\delta_{x}$ denotes the Dirac measure. It is stationary and ergodic with the invariant distribution

$$
\pi(d x)=(\alpha-1) /\left(2|x|^{\alpha}\right) d x \text { for }|x|>1 .
$$

Zhao et al. (2010) showed that $S_{n}=\sum_{i=1}^{n} \operatorname{sign}\left(X_{i}\right)$ does not satisfy the central limit theorem under any normalization. In addition they $\operatorname{showed}$ that $\operatorname{var}\left(S_{n}\right) \sim$ $c n^{2 / \alpha}$. For statistical inference of this example, we can use the CLT given in our Theorem 1 immediately as $h_{n} n^{2 / \alpha-1} \rightarrow 0$.

\section{Conclusion and remarks}

In this paper we propose a method for constructing confidence intervals for the mean or for testing statistical hypotheses for the mean of a dependent stationary sequence with finite second moment. The method is robust in the sense that we do not impose a specific restriction on the dependence structure of the sequence except for the ergodicity and the consistency of the sample mean in $L_{2}$. The estimator we propose is $\hat{r}_{n}$, defined by (3) leading to the confidence intervals defined by (6). For applications, it is convenient to use a kernel $K$ following a standard normal distribution and to generate $\left(X_{i} ; 1 \leq i \leq n\right)$ also from a standard normal variable. For this choice of $f$ and $K$, we obtained $f(0)=$ $1 / \sqrt{2 \pi}, \int K^{2}(u) d u=1 /(2 \sqrt{\pi}), \int u^{2} K(u) d u=1, f^{\prime \prime}(0)=-1 / \sqrt{2 \pi}$. Thus, the plug in estimator of the optimal bandwidth is

$$
h_{o}=\left(\frac{\overline{Y^{2}}}{n \sqrt{2} \bar{Y}^{2}}\right)^{0.2}, \text { provided } \operatorname{var}\left(\bar{Y}_{n}\right)=o\left(n^{-0.8}\right) .
$$

The $(1-\alpha) 100 \%$ confidence interval for $\mu_{Y}$ becomes

$$
\left(\hat{r}_{n}-z_{\alpha / 2} \sqrt{\frac{1}{2 h_{n} n^{2}} \sum_{i=1}^{n} Y_{i}^{2}}, \hat{r}_{n}+z_{\alpha / 2} \sqrt{\frac{1}{2 h_{n} n^{2}} \sum_{i=1}^{n} Y_{i}^{2}}\right),
$$

where

$$
\hat{r}_{n}=\frac{1}{n h_{n}} \sum_{i=1}^{n} Y_{i} \exp \left[-\left(\frac{1}{h_{n}} X_{i}\right)^{2}\right] .
$$

It is easy to see that the size of the confidence interval depends on the $\operatorname{var}\left(\bar{Y}_{n}\right)$ via condition (5), which restricts the size of $n h_{n}$. The larger $\operatorname{var}\left(\bar{Y}_{n}\right)$, the larger the size of the interval.

Our result is asymptotic. We have conducted a numerical study to test the performance of the confidence intervals based on formula (21) on finite sample sizes. We have constructed confidence intervals based on samples from an ARFIMA $(0, d, 0)$ with innovations $\left(\xi_{j}\right)_{j \in Z}$. In our simulations we vary the size of $d$, which controls the dependence strength, and accordingly the size of $h_{n}$. Since the second moment of $Y$ is important we also vary the distribution of 
$Y$ by considering various distributions for the innovations. In all the situations, for relatively large sample size, our methods returned reliable results.

Based on standard normal innovations we simulated an $\operatorname{ARFIMA}(0, .09,0)$ sequence $\left(Y_{n}^{\prime}\right)$ and set $Y_{n}=3+Y_{n}^{\prime}$. For a sample size $n=100$, and using optimal bandwidth, we found that a $95 \%$ confidence for $\mu_{Y}$ is $(2.9,3.49)$, while for $n=1000$ the $95 \%$ confidence for $\mu_{Y}$ is $(2.81,3.04)$.

From 100 confidence intervals constructed this way, 96 of them covered the real mean, which turns to be a statistically significant result for $95 \%$ confidence intervals. Similar results were obtained for simulations based on $\operatorname{ARFIMA}(0, .09,0)$ with uniform innovations $U(-0.5,0.5)$ and with centered $\chi^{2}(2)$ innovations.

When $d>0.1$, according to (20), $h_{o}$ does not satisfies the restriction and we selected instead $h_{n}=n^{-2 d}$. For this case we simulated samples from $\operatorname{ARFIMA}(0, .49,0)$ and standard normal innovations, $n=500$. We obtained that from 100 such simulations, 92 of $90 \%$ confidence intervals covered the mean.

Acknowledgement. The first author was supported by the College of Liberal Arts Summer grant and the second author was partially supported by the NSF grant DMS-1512936 and a grant from the Taft Research Center.

\section{References}

[1] Billingsley, P. (1995). Probability and measure. 3-rd edition, John Willey \& Sons.

[2] Billingsley, P. (1999). Convergence of probability measures. 2-nd edition, John Willey \& Sons.

[3] Bradley, R. (1983). Asymptotic normality of some kernel-type estimators of probability density. Statist. Probab. Lett. 1, 295-300.

[4] Bosq, D. (1996). Nonparametric statistics for stochastic processes: estimation and prediction. New York: Springer-Verlag.

[5] Bosq, D.,Merlevède, F. and Peligrad, M. (1999). Asymptotic normality for density kernel estimators in discrete and continuous time. Journal of Multivariate Anal. 68, 79-95.

[6] Collomb, G. (1984). Propriétés de convergence presque complète du prédicteur à noyau. Z. Wahr. Verwandte Gebiete 66, 441-460.

[7] Granger, C. W. and Joyeux, R. (1980). An introduction to long-memory time series models and fractional differencing. J. Time Ser. Anal 1, 15-29.

[8] Härdle, W. (1991). Smoothing Techniques With Implementation in S. Springer-Veralg

[9] Hong, S. Y. and Linton, O. (2016). Asymptotic properties of a Nadaraya-Watson type estimator for regression functions of infinite order. arXiv:1604.06380 
[10] Hosking, J. R. M. (1981). Fractional differencing. Biometrika 68, 165-176.

[11] Jones, M.C., Marron, J.S. and Sheather, S.J. (1996). A brief survey of bandwidth selection for density estimation. Journal of the American Statistical Association 91, 401-407.

[12] Nadaraya, E.A. (1964). On estimating regression. Theory of Probability \& Its Applications, 9, 141-142.

[13] Long, H. and Qian, L. (2013). Nadaraya-Watson estimator for stochastic processes driven by stable Lévy motions. Electronic Journal of Statistics 7, $1387-1418$.

[14] Peligrad, M. (1992). Properties of uniform consistency of the kernel estimators of density and of regression functions under dependence assumptions. Stochastics and Stochastic Reports 40, 147-168.

[15] Peligrad M. (1998.) On the blockwise bootstrap for empirical processes for stationary sequences. Annals of Probability 26, 877-901.

[16] Wang, Q., Lin, X-Y. and Gulati, C. M. (2001). Asymptotics for moving average processes with dependent innovations. Stat. and Probab. Letters. 54, 347-356.

[17] Watson, G.S. (1964). Smooth regression analysis. Sankhya Series A, 26, 359-372.

[18] Yoshihara, K. (1994). Weakly Dependent Stochastic Sequences and their Applications: Volume IV: Curve Estimation Based on Weakly Dependent Data. Sanseido, Tokyo, Japan.

[19] Zhao, O., Woodroofe, M. and Volný, D. (2010). A central limit theorem for reversible processes with nonlinear growth of variance. J. Appl. Prob. 47, 1195-1202. 